\title{
Stability and angular-momentum transport of fluid flows between corotating cylinders
}

\author{
M. Avila \\ Max Planck Institute for Dynamics and \\ Self-Organization (MPIDS), 370r77 Göttingen, Germany \\ Institute of Fluid Mechanics, Friedrich-Alexander-Universität Erlangen-Nürnberg, \\ Cauerstraße 4, 91058 Erlangen, Germany
}

(Dated: September 10, 2018)

\begin{abstract}
Turbulent transport of angular momentum is a necessary process to explain accretion in astrophysical disks. Although the hydrodynamic stability of disk-like flows has been tested in experiments, results are contradictory and suggest either laminar or turbulent flow. Direct numerical simulations reported here show that currently investigated laboratory flows are hydrodynamically unstable and become turbulent at low Reynolds numbers. The underlying instabilities stem from the axial boundary conditions, affect the flow globally and enhance angular momentum transport.
\end{abstract}

PACS numbers: 47.20.-k,95.30.Lz,47.54.-r 
Accretion in astrophysical disks requires the flow of mass towards a central gravitating body. The ensuing loss of momentum must be balanced by outward angular momentum transfer among gas particles [1]. If the motion of orbiting gas was laminar molecular transport would be orders of magnitude too slow for accretion to take place, and so considering a turbulent viscosity becomes necessary [2]. However, in Keplerian disks the gas rotates as $\Omega \propto r^{-3 / 2}$ and laminar motion is linearly stable according to the Rayleigh criterion. Although axial magnetic fields can drive turbulence via the magnetorotational instability [3] , it is not clear whether this operates in weakly ionized disks. On the other hand, it is well known that linearly stable shear flows (such as pipe flow) can become turbulent due to finite amplitude disturbances. Whether Keplerian flows are susceptible to such a transition scenario or remain stable despite the large Reynolds numbers $(R e)$, is a topic of great interest and the source of much controversy [4].

The stability of disk-like flows is typically probed in laboratory experiments of fluid between two concentric and independently rotating cylinders, Taylor-Couette flow (TCF). In the infinite-cylinder idealization, the Navier-Stokes equations admit a pure rotary solution

$$
\Omega(r)=\frac{\Omega_{2} r_{2}^{2}-\Omega_{1} r_{1}^{2}}{r_{2}^{2}-r_{1}^{2}}+\frac{\left(\Omega_{1}-\Omega_{2}\right)\left(r_{1} r_{2}\right)^{2}}{r_{2}^{2}-r_{1}^{2}} \frac{1}{r^{2}},
$$

commonly referred to as Couette flow. Here $r_{1}$ and $r_{2}$ are the radii of the inner and outer cylinders and $\Omega_{1}$ and $\Omega_{2}$ their angular velocities. When $\left(r_{1} / r_{2}\right)^{2}<\Omega_{2} / \Omega_{1}<1$ the angular velocity decreases outward but the angular momentum increases (quasi-Keplerian flows). Accretion disks are stratified in the axial direction and are thus best modelled considering an unbounded domain, thereby avoiding artificial boundary conditions [5]. Most experiments and simulations focus, however, on the physics of the disk's midplane and neglect stratification. Under this assumption simulations typically employ axially periodic boundary conditions, whereas in experiments cylinders have a finite-length $h$. Hence the degree to which (1) may be approximated is compromised by the axial boundary conditions and length-to-gap aspect-ratio $\Gamma=h /\left(r_{2}-r_{1}\right)$. In particular, solid axial boundaries result in a basic state with nonzero radial and axial velocity components (Ekman flow). Hence, producing Couette-like profiles in experiments poses an extraordinary challenge, which may be addressed by considering very tall cylinders or splitting the endwalls in several rings that rotate at independent angular speeds [6]. The latter strategy has been implemented in the Princeton Taylor-Couette experiment [7], which has a short aspect-ratio $\Gamma=2.104$ but 
whose endwalls are split into two independently rotating rings. From simultaneous Laser Doppler Velocity measurements of azimuthal and radial velocity components, Ji et al. [8] suggest that quasi-Keplerian flows at Reynolds numbers up to millions are essentially laminar. From this, they conclude that purely hydrodynamic mechanisms cannot transport angular momentum at the rates required for accretion to occur in disks.

This conclusion has been recently challenged in a new experimental study by Paoletti and Lathrop [9], who report from direct torque measurements at the inner cylinder that Keplerian flows at $R e \gtrsim 10^{6}$ are fully turbulent. When extrapolated to astrophysical disks, their results indicate that transport occurs at accretion relevant rates, in agreement with previous estimations [10]. Despite having tall cylinders $\Gamma=11.47$, the Maryland experiment has solid endwalls that are attached to the outer cylinder and hence cannot be rotated independently. Although these boundary conditions are known to generate vigorous Ekman vortices and greatly increase the exerted torque, their contribution is discarded by dividing the inner cylinder into three sections and measuring torque only in the central one. Despite efforts in the Princeton and Maryland experimental setups to mitigate endwall effects it is, however, unclear whether their results can be used to infer the stability of flows in astrophysical disks [4]. In this Letter it is shown that current laboratory experiments of quasi-Keplerian flows become turbulent already at $R e=O\left(10^{3}\right)$ due to hydrodynamic instabilities stemming from the axial boundary conditions. Moreover, it is found that turbulence fills the entire flow domain and as a result the momentum transfer is globally enhanced.

Here direct numerical simulations of flows with the precise geometry and boundary conditions of the Princeton and Maryland experiments were performed. The Navier-Stokes equations for an incompressible Newtonian fluid of velocity $\mathbf{v}$

$$
\partial_{t} \mathbf{v}+(\mathbf{v} \cdot \nabla) \mathbf{v}=-\nabla p+\Delta \mathbf{v}, \quad \nabla \cdot \mathbf{v}=0
$$

were rendered dimensionless by scaling lengths and time with the gap-width $d=r_{2}-r_{1}$ and viscous time $d^{2} / \nu$, where $\nu$ is the kinematic viscosity of the fluid. The solution of (2) was formulated in primitive variables in cylindrical coordinates $(r, \theta, z)$ and a second-order time-splitting method with consistent boundary conditions for the pressure was used [11]. The spatial discretization consists of Chebyshev collocation in $(r, z)$ and a Galerkin-Fourier expansion in $\theta$. The code converges spectrally in the three directions [12] and was validated against a Legendre-Fourier-Galerkin code [13]. Here the resolution was chosen to ensure 
that computed torque values were accurate to at least $1 \%$.

The geometry of the Taylor-Couette system is specified by the radii-ratio $\eta=r_{1} / r_{2}$ and the length-to-gap aspect-ratio $\Gamma$. The dimensionless boundary conditions at the cylinders $\operatorname{read}\left(v_{r}, v_{\theta}, v_{z}\right)\left[r_{1,2}, \theta, z\right]=\left(0, R e_{1,2}, 0\right)$, where $R e_{1}=d r_{1} \Omega_{1} / \nu\left(R e_{2}=d r_{2} \Omega_{2} / \nu\right)$ is the inner (outer) cylinder Reynolds number. Because of differential rotation the angular velocity changes abruptly at adjacent rotating boundaries. In the Princeton experiment, the endwall is split at mid-radius $r_{m}=\left(r_{1}+r_{2}\right) / 2$ into two independently rotating rings. Hence, there are four independent angular speeds; $\Omega_{1}$ and $\Omega_{2}$, for inner and outer cylinder, and $\Omega_{3}$ and $\Omega_{4}$, for inner and outer rings. To preserve spectral convergence discontinuities in angular velocity were regularized, yielding the following boundary condition at the endwalls $z= \pm \Gamma / 2$

$$
\begin{aligned}
\Omega(r)= & \left(\Omega_{1}-\Omega_{3}\right) \exp \left[-\left(r-r_{1}\right) / \epsilon\right]+ \\
& \left(\Omega_{2}-\Omega_{4}\right) \exp \left[-\left(r_{2}-r\right) / \epsilon\right]+ \\
& \frac{\Omega_{3}+\Omega_{4}}{2}+\frac{\Omega_{4}-\Omega_{3}}{2} \tanh \left[\left(r-r_{m}\right) / \epsilon\right],
\end{aligned}
$$

with $\epsilon \in\left[5 \times 10^{-3}, 10^{-2}\right]$ (see Ref. 14). The boundary condition modeling the Princeton experiment [8] is shown as circles in Fig. 1 1 a. Due to the sharp gradient $\partial \Omega /\left.\partial r\right|_{r_{m}}$ and the clustering of Chebyshev points close to the boundaries, a large number of radial points $\left(n_{r}=351\right)$ was required to accurately simulate the split endwall. In the axial and azimuthal directions up to $n_{z}=281$ Chebyshev points and $n_{\theta}=256$ Fourier modes were used. The Maryland experiment has a single solid ring attached to the outer cylinder $\left(\Omega_{4}=\Omega_{3}=\Omega_{2}\right)$ and there is only a strong gradient at $r_{1}$ (see crosses in Fig. 17a). Here up to $n_{z}=601$, $n_{\theta}=384$ and $n_{r}=61$ were used.

To put TCF in the wider context of rotating shear flows it is useful to define a shear Reynolds number $R e=2 /(1+\eta)\left|R e_{2} \eta-R e_{1}\right|$ and a rotation number $R_{\Omega}=(1-\eta)\left(\operatorname{Re}_{1}+\right.$ $\left.R e_{2}\right) /\left(R e_{2} \eta-R e_{1}\right)$, which measure the ratio of shear to viscous forces and the ratio of mean rotation to shear [15], respectively. Here the sign of $R_{\Omega}$ distinguishes between cyclonic $\left(R_{\Omega}>0\right)$ and anticyclonic flows $\left(R_{\Omega}<0\right)$, with $-2<R_{\Omega}<-1$ corresponding to quasiKeplerian rotation. In the experiments of Ji et al. [8] $R_{\Omega}=-1.038$ and $\eta=0.3478$, and the same values were used here, whereas Lathrop and Paoletti [9] have systematically studied both cyclonic and anticyclonic regimes at $\eta=0.7245$. Here, $\eta=0.7245$ and the value $R_{\Omega}=$ -1.047 was chosen (corresponding to their Rossby number $R o=R e_{1} /\left(\eta R e_{2}\right)-1=0.85$ ).

The endwall influence in the Maryland experiment is illustrated in Fig. 1b, showing the 

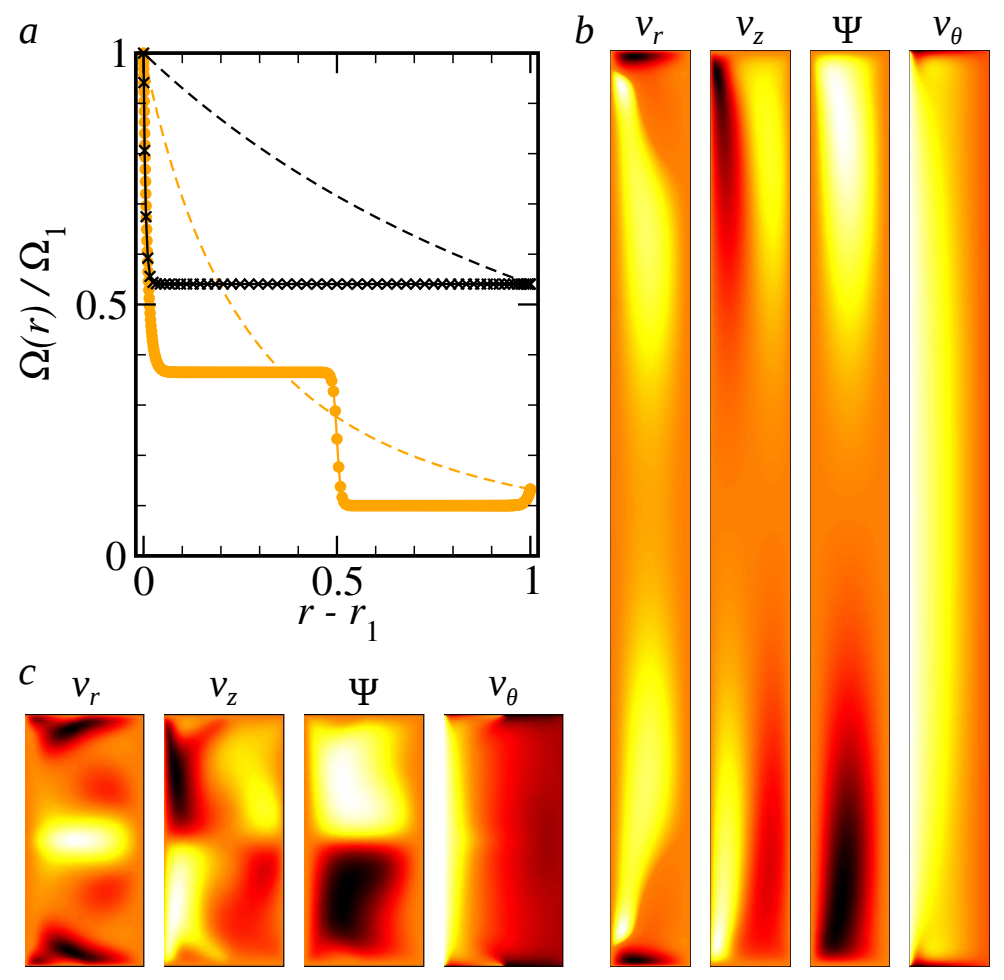

FIG. 1. (color online) (a) Angular velocity at the endwalls for the Princeton (circles) and Maryland (crosses) experiments, and Couette flow (dashed lines). $(b-c)$ Steady basic states at $R e=320$ (Maryland) and $R e=772$ (Princeton). White (black) corresponds to maximum (minimum) velocity and the radial direction is horizontal with left (right) corresponding to inner (outer) cylinder.

velocity field of the steady basic state at $R e=320$. At the endwalls there is a strong negative radial velocity inflow, which generates axial velocities pointing towards mid-height along the inner cylinder and result in an axially dependent azimuthal velocity. Figure 1 $c$ shows the basic state of the Princeton experiment at $R e=772$. Because of the small aspect-ratio the meridional circulation generates a strong radial outward flow at mid-height that increases the outward transport of azimuthal velocity. Were the angular speeds of the endwall rings selected according to the ideal Couette profile [6, 16] instead of the values used in experiments [8] and reproduced here, profiles with weaker meridional circulation and hence closer to Couette flow could be obtained. This approach was used in previous numerical simulations of the Princeton configuration [17].

The visualizations of Fig. $1 b-c$ hint at the difficulty of realizing quasi-Keplerian profiles in a laboratory experiment even at very low Reynolds numbers. The endwall boundary conditions change the velocity field not just locally but globally across the domain. In fact, 

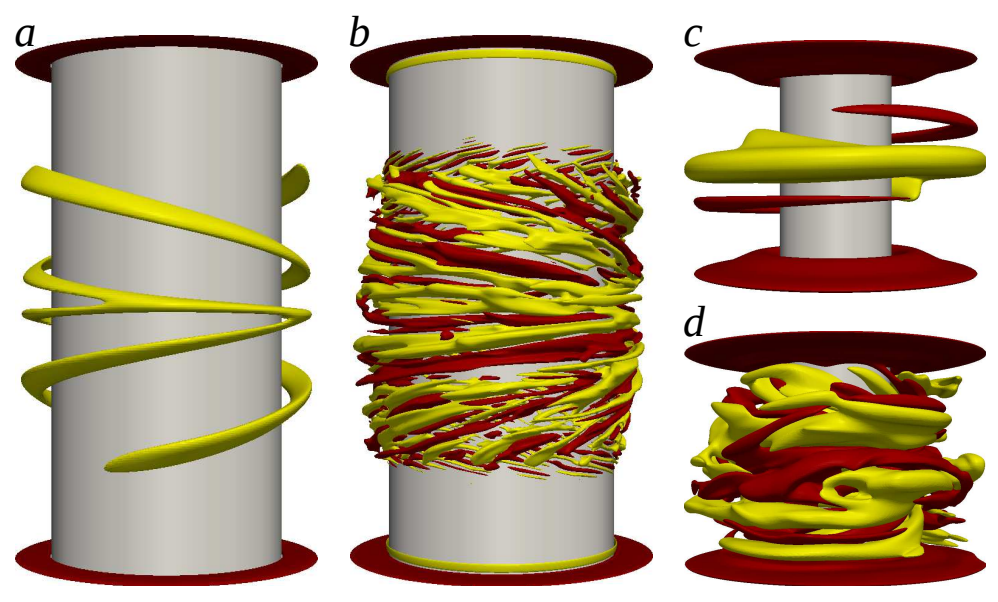

FIG. 2. (color online) Three-dimensional view of isosurfaces of negative (red) and positive (yellow) radial velocity. (a) $m=2$ rotating wave at $R e=1332$ and $(b)$ turbulent flow at $R e=5328$ from simulations of the Maryland experiment. (c) Modulated rotating wave with $m=1$ and $m=2$ at $R e=1545$ and $(d)$ turbulent flow at $R e=6437$ from simulations of the Princeton experiment.

at slightly higher Reynolds numbers the flow becomes three-dimensional and time-dependent via supercritical Hopf bifurcations. In the Maryland configuration instability occurs first at $R e_{c}=352$ to prograde rotating waves with azimuthal wavenumber $m=5$ and localized at the endwalls. Beyond $R e_{c}$ multiplicity of states, with different symmetries and wavenumber $m \in[2,5]$, was found, whereas for $R e \gtrsim 1330$ only a global $m=2$ mode remained stable and was obtained regardless of initial conditions (see Fig. $2 a$ ). Further increasing the Reynolds number led to modulated waves and a quick transition to temporal chaos at about $R e \simeq 1600$. Subsequently, spatial periodicity was lost and the spectra broadens as the flow became gradually turbulent; Fig. $2 b$ shows a flow snapshot at $R e=5328$. This transition picture is also representative of the simulations of the Princeton experiment. Here the basic steady state becomes unstable at $R e=1448$ almost simultaneously to $m=1$ and $m=2$ rotating waves, which were found to coexist in space and time (see Fig. 2r). By no means is this situation generic: changing the relative rotation of the cylinders one of $m=1,2$ was found to bifurcate first. Further increasing the Reynolds number led to very complex and strongly three-dimensional flow as shown in Fig. $2 d$.

The stability of quasi-Keplerian TCF with endwalls attached to the outer cylinder and $\eta=0.7245$ is shown in Fig. $3 a$. The minimum critical Reynolds number is attained at the Rayleigh line $\left(R_{\Omega}=-1\right)$ and increases as differential rotation decreases, but with $R e_{c}<10^{4}$ 

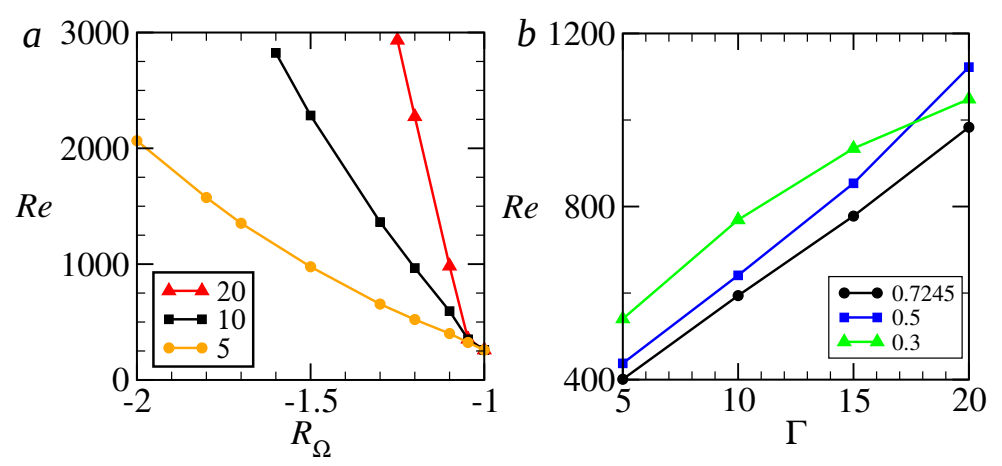

FIG. 3. (color online) Stability curves of quasi-Keplerian Taylor-Couette flows with endwalls attached to the outer cylinder: $(a) \eta=0.7245$ and $\Gamma$ as in the legend, $(b) R_{\Omega}=-1.1$ and $\eta$ as in the legend.

across the whole quasi-Keplerian regime. Close to the Rayleigh line modes localized to the endwalls bifurcate first, whereas for $R_{\Omega} \lesssim-1.1$ global instability modes (as in Fig. $2 a$ ) dominate. The former are similar to those observed experimentally in Ref. 18 and the latter are similar to those reported by Avila et al. [12], who studied global boundary layer effects on flows between exactly co-rotating cylinders and stationary endwalls. Figure $3 b$ further shows that endwall instabilities depend weakly on geometry and hence generically govern the dynamics of quasi-Keplerian TCF. It is worth noting that endwall instabilities persist beyond the Rayleigh line and coexist with Taylor vortices close to the onset of centrifugal instability.

The onset of hydrodynamic instability and transition to turbulence are expected to radically change the radial transport of azimuthal momentum. The solid lines in Fig. 四 show normalized average azimuthal velocity profiles $\left\langle v_{\theta}\right\rangle_{\theta, t} /\left(r_{1} \Omega_{1}\right)$ at mid-height for simulations of the Maryland experiment at $R e=5328$ (black solid line) and Princeton experiment at $R e=6437$ (gray solid line, orange online). At the inner cylinder the profiles are steeper than laminar Couette flow (dashed lines), implying larger torques on the cylinder surface. It is worth noting that in TCF between infinite cylinders the transverse current of azimuthal motion $J^{\Omega}=r^{3}\left[\left\langle v_{r} \Omega\right\rangle_{\theta, z, t}-\nu \partial_{r}\langle\Omega\rangle_{\theta, z, t}\right]$ is a conserved quantity [19], and as a consequence the dimensionless torque $G=\nu^{-2} J^{\Omega}$ is the same at inner and outer cylinder. This does not hold, however, for flows confined by no-slip axial boundaries. Torque profiles along the inner cylinder, normalized with the laminar Couette torque, are shown in Figure $4 \mathrm{~b}$. Because of the sharp change in $\Omega$ occurring across a small gap between inner cylinder and endwalls 


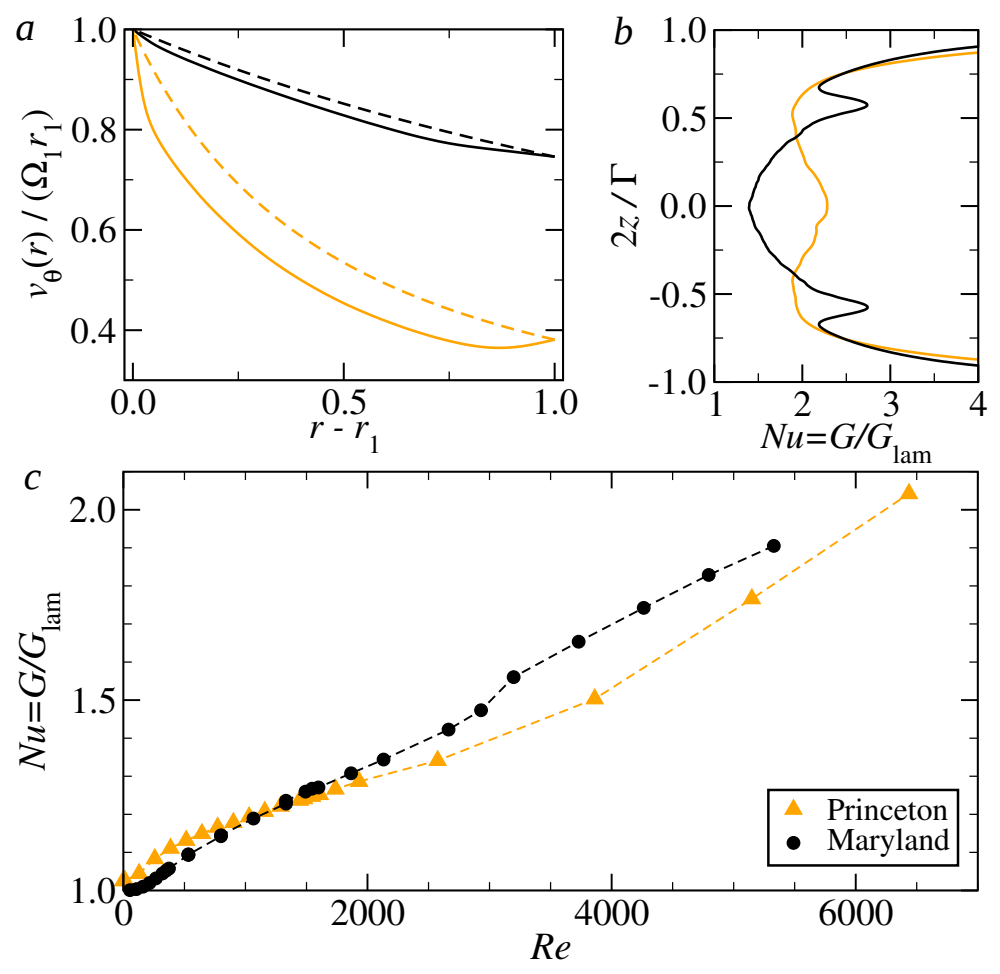

FIG. 4. (color online) (a) Average azimuthal velocity at mid-height for simulations of the Maryland ( $R e=5328$, black solid line) and Princeton $(R e=6437$, orange solid line $)$ experiments. The dashed lines are laminar Couette flow. (b) Torque $N u=G / G_{\text {lam }}$ along the inner cylinder, solid lines as in $(a)$. (c) Reynolds number dependence of the torque on the central section of the inner cylinder $(0.6>|2 z / \Gamma|$ and $0.4>|2 z / \Gamma|$ for simulations of the Maryland and Princeton experiments).

(see Fig. 1 1 ), the torque required to rotate the inner cylinder faster than the endwall is very large. This increase in local torque as the endwalls $2 z / \Gamma= \pm 1$ are approached can be seen in Figure $4 b$. Although the direct contribution of the endwall is largely avoided by the measurement technique in the experiments, the torque in the central section remains well above laminar because of turbulent fluctuations.

The simulations of the Maryland experiment show a clear change in the torque behavior at about $R e=3000$ (see black curve in Fig. 4k). This is related to the appearance of the two torque peaks at $2 z / \Gamma \sim \pm 0.5$ in Fig. $4 b$ and is caused by the onset of small-scale vortices which have opposite spiral orientation to the structure of the primary rotating wave (see Fig $2 b$ ). In both experiments the torque has already doubled the laminar value at $R e \sim 6000$ due to the endwall driven instabilities.

In spite of the disparity in Reynolds numbers, it is tempting to compare these numerical 
results to experimental observations. Van Gils et al. [20] have measured torque at $R e>10^{5}$ and have observed an effective universal scaling law $N u=G / G_{\text {lam }}=a\left(R_{\Omega}\right) R e^{0.76}$ that holds throughout the linearly unstable regime of TCF. Surprisingly, Paoletti and Lathrop [9] (see also Ref. 21) have demonstrated that this law applies to linearly stable cyclonic and anti-cyclonic Rayleigh-stable regimes as well, that is including quasi-Keplerian rotation. It is then natural to ask how this universal behavior connects to the complex flows uncovered in this work. It is speculated here that a transition between endwall-driven turbulence to the universal $N u \propto R e^{0.76}$ scaling reported in experiments may take place at intermediate $R e$. Interestingly, in the case of stationary outer cylinder a cross-over marking the transition from centrifugally to shear-driven turbulence at $R e \simeq 13000$ was reported [22]. If an analogous cross-over was found in quasi-Keplerian flows and shown to be independent of aspect-ratio and endwall boundary condition, a strong case for the existence of hydrodynamic turbulence in astrophysical disks would be made. In fact, ingredients of shear-driven turbulence such as transient growth of disturbances are found also in quasi-Keplerian flows, although significantly only at $R e=O\left(10^{6}\right)$ [23]. On the other hand, it would be interesting to investigate the connection between the complex flows found here and the quiescent flows reported by Ji et al. [8] at large $R e$.

In conclusion, current laboratory experiments designed to approximate flow profiles expected from accretion disks become turbulent at moderate Reynolds number due to imposed boundary conditions. Although these instabilities are generic and hence cannot possibly be avoided, universal scaling suggests that shear mechanisms might overwhelm endwall effects at large Reynolds number. In order to probe this hypothesis new experiments with variable aspect ratio and different axial boundary conditions should be conducted. These would provide great indsight on the physical mechanisms of rotating shear flows and might shed light on the origin of turbulence in astrophysical disks.

\section{ACKNOWLEDGMENTS}

Support from the Max Planck Society is acknowledged and the Engineering and Physical Sciences Research Council (Grant No. EP/F017413/2) is acknowledged. The author is 
grateful to Kerstin Avila and Bjoern Hof for discussions.

[1] J. Pringle, Ann. Rev. Astron. Astrophys. 19, 137 (1981)

[2] N. Shakura and R. Sunyaev, Astron. Astrophys. 24, 337 (1973)

[3] S. A. Balbus and J. F. Hawley, Rev. Mod. Phys. 70, 1 (1998)S. A. Balbus 41, 555 (2003)

[4] S. A. Balbus, Nature 470, 475 (2011)

[5] J. A. Barranco and P. S. Marcus, Astrophys. J. 623, 1157 (2005)

[6] R. Hollerbach and A. Fournier, AIP Conf. Proc., 114(2004)

[7] E. Schartman, H. Ji, and M. J. Burin, Rev. Sci. Instrum. 80, 024501 (2009)

[8] H. Ji, M. Burin, E. Schartman, and J. Goodman, Nature 444, 343 (2006)

[9] M. S. Paoletti and D. P. Lathrop, Phys. Rev. Lett. 106, 024501 (2011)

[10] D. Richard and J. P. Zahn, Astron. Astrophys. 347, 734 (1999)

[11] S. Hughes and A. Randriamampianina, Intnl J. Num. Meth. Fluids 28, 501 (1998)I. Mercader, O. Batiste, and A. Alonso, Computers \& Fluids 39, 215 (2010)

[12] M. Avila, M. Grimes, J. M. Lopez, and F. Marques, Phys. Fluids 20, 104104 (2008)

[13] F. Marques and J. M. Lopez, J. Fluid Mech. 561, 255 (2006)

[14] J. M. Lopez and J. Shen, J. Comp. Phys. 139, 308 (1998)

[15] B. Dubrulle, O. Dauchot, F. Daviaud, P. Y. Longaretti, D. Richard, and J. P. Zahn, Phys. Fluids 17, 095103 (2005)

[16] A. Kageyama, H. Ji, J. Goodman, F. Chen, and E. Shoshan, J. Phys. Soc. Japan 73, 2424 (2004)

[17] A. Obabko, F. Cattaneo, and P. Fischer, Physica Scripta T132, 014029 (2008)

[18] C. D. Andereck, S. S. Liu, and H. L. Swinney, J. Fluid Mech. 164, 155 (1986)

[19] B. Eckhardt, S. Grossmann, and D. Lohse, J. Fluid Mech. 581, 221 (2007)

[20] D. P. M. van Gils, S. G. Huisman, G. W. Bruggert, C. Sun, and D. Lohse, Phys. Rev. Lett. 106, $24502(2011)$

[21] M. Paoletti, D. van Gils, B. Dubrulle, C. Sun, D. Lohse, and D. Lathrop, Arxiv preprint arXiv:1111.6915(2011)

[22] D. P. Lathrop, J. Fineberg, and H. L. Swinney, Phys. Rev. Lett. 68, 1515 (1992)G. S. Lewis and H. L. Swinney, Phys. Rev. E 59, 5457 (1999) 
[23] P. A. Yecko, Astron. Astrophys. 425, 385 (2004) 